\title{
Inelastic Scattering of Photon Pairs in Qubit Arrays with Subradiant States
}

\author{
Yongguan Ke, ${ }^{1,2}$ Alexander V. Poshakinskiy, ${ }^{3}$ Chaohong Lee $\odot,{ }^{1,4, *}$ \\ Yuri S. Kivshar $\odot^{2,5}$ and Alexander N. Poddubny ${ }^{2,3,5, \uparrow}$ \\ ${ }^{1}$ Guangdong Provincial Key Laboratory of Quantum Metrology and Sensing \& School of Physics and Astronomy, \\ Sun Yat-Sen University (Zhuhai Campus), Zhuhai 519082, China \\ ${ }^{2}$ Nonlinear Physics Centre, Research School of Physics, Australian National University, \\ Canberra, Australian Capital Territory 2601, Australia \\ ${ }^{3}$ Ioffe Institute, St. Petersburg 194021, Russia \\ ${ }^{4}$ State Key Laboratory of Optoelectronic Materials and Technologies, Sun Yat-Sen University (Guangzhou Campus), \\ Guangzhou 510275, China \\ ${ }^{5}$ ITMO University, St. Petersburg 197101, Russia
}

(Received 19 August 2019; published 19 December 2019)

\begin{abstract}
We develop a rigorous theoretical approach for analyzing inelastic scattering of photon pairs in arrays of two-level qubits embedded into a waveguide. Our analysis reveals a strong enhancement of the scattering when the energy of incoming photons resonates with the double-excited subradiant states. We identify the role of different double-excited states in the scattering, such as superradiant, subradiant, and twilight states, as a product of single-excitation bright and subradiant states. Importantly, the $N$-excitation subradiant states can be engineered only if the number of qubits exceeds $2 N$. Both the subradiant and twilight states can generate long-lived photon-photon correlations, paving the way to storage and processing of quantum information.
\end{abstract}

DOI: 10.1103/PhysRevLett.123.253601

Introduction.-Nonlinear manipulation of light via its interaction with matter plays an essential role in optics and its applications [1-3], including optical communications [4] and sensing [5]. The light-matter interaction can be strongly modified by collective coherent superradiance or subradiance, where the spontaneous emission speeds up or slows down [6-9]. Both superradiance and subradiance have been realized in various systems [10-24], and they provide novel opportunities to explore the interplay between collective excitations in materials and nonlinear effects in scattering of light $[7,8]$. Compared to superradiance, subradiance enables a longer time for light-matter interaction and giant nonlinear response [25-27]. To the best of our knowledge, the enhancement of light-matter interaction by subradiant modes has been explored mostly in classical optics.

It is appealing and challenging to exploit the quantum nonlinearities at a few-photon level $[1,2,7,8]$. One of the simplest nonlinear quantum processes is the inelastic scattering of photon pairs. It exists in waveguides coupled to a single qubit or qubit arrays, see Fig. 1, and is sensitive to the two-photon bound states [28-31]. The scattering is greatly enhanced when an incoming or outgoing individual photon excites a single-particle subradiant state [31-33]; the concept of multiexcitation subradiant states has been put forward $[32,34,35]$. It has been predicted that the subradiant mode has a fermionic character and a decay rate with cubic suppression in the number of qubits [34-36]. However, the role of collective many-body mechanisms in the enhancement of quantum nonlinear processes remains unclear.
In this Letter, we reveal that many-body subradiant states can enhance the incoherent scattering of photon pairs in arrays of two-level qubits supporting long-lived photonphoton correlations. Specifically, we demonstrate sharp scattering resonances when the energy of the two-particle subradiant state matches the total energy of photon pairs [28,29,37-41]. Importantly, considered resonances are not affected by the destructive quantum interference known to suppress two-photon scattering [28,42]. The $N$-particle subradiant states appear only in periodic arrays with at least $2 N$ qubits; e.g., the two-particle state requires at least four qubits. It is also possible to realize a resonant condition for single- and double-excited subradiant states simultaneously. We develop a matrix formulation for the rigorous Green's function technique valid for an arbitrary arrangement of qubits. This allows us to analytically identify the role of different double-excited states in the scattering and classify them by the coupling strength. In addition to the double-excited superradiant and subradiant states, we

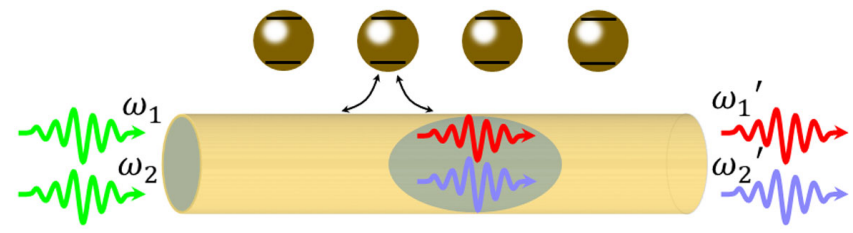

FIG. 1. Schematic illustration of the photon pairs propagating along a waveguide with a qubit array and exhibiting inelastic scattering. 
introduce a new concept of twilight state, which is a product of single-excited bright and subradiant states. Our results demonstrate that the coupling of light to quantum matter is far from being fully understood even for the classical Dicke model, and thus this opens a new avenue for manipulating quantum interactions, correlations, and entanglement.

Model.-We consider the system shown schematically in Fig. 1. It consists of $N$ periodically spaced qubits, coupled to $M$ photons in the one-dimensional waveguide, and it is characterized by the Hamiltonian

$$
\begin{aligned}
H= & \sum_{k} \hbar \omega_{k} a_{k}^{\dagger} a_{k}+\sum_{j} \hbar \omega_{0} b_{j}^{\dagger} b_{j}+\frac{\hbar \chi}{2} \sum_{j} b_{j}^{\dagger} b_{j}^{\dagger} b_{j} b_{j} \\
& +\frac{\hbar g}{\sqrt{L}} \sum_{j, k}\left(b_{j}^{\dagger} a_{k} e^{i k z_{j}}+b_{j} a_{k}^{\dagger} e^{-i k z_{j}}\right) .
\end{aligned}
$$

Here, $a_{k}$ are the annihilation operators for the waveguide photons with the wave vectors $k$ (the corresponding frequencies are given by $\omega_{k}=c|k|$ with the light velocity $c$ ), $g$ is the interaction constant, $L$ is the normalization length, and $b_{j}$ are the (bosonic) annihilation operators for the qubit excitations with the frequency $\omega_{0}$, located at the point $z_{j}$. In Eq. (1), we consider the general case of anharmonic multilevel qubits; the two-level case can be obtained in the limit of large anharmonicity $(\chi \rightarrow \infty)$ where the multiple occupation is suppressed $[43,44]$. The photons can be traced out in Eq. (1), yielding an effective model for describing the excitations in the qubits $[34,45]$,

$$
\mathcal{H}=\sum_{i, j} H_{i, j}^{(1)}\left(\omega_{0}\right) b_{i}^{\dagger} b_{j}+\frac{\hbar \chi}{2} \sum_{j} b_{j}^{\dagger} b_{j}^{\dagger} b_{j} b_{j},
$$

where

$H_{i j}^{(1)}(\omega) \equiv \hbar \omega_{0} \delta_{i j}-i \hbar \Gamma_{0} e^{i \omega / c\left|z_{i}-z_{j}\right|}, \quad i, j=1, \ldots, N$.

The Hamiltonian Eq. (3) is non-Hermitian, and it takes into account the radiative losses characterized by the radiative decay rate for a single qubit in a waveguide, $\Gamma_{0}=g^{2} / c$. The interaction between the qubits is long-ranged since it is mediated by the photons propagating in the waveguide. We assume that the spacing between the qubits is small enough so that the non-Markovian Hamiltonian (3) with the phases $(\omega / c)\left|z_{i}-z_{j}\right|$ can be replaced by $H_{i j}^{(1)}\left(\omega_{0}\right)$ [47]. From now on, we neglect the non-Markovian effects [43].

Double-excited states.-Before proceeding to the study of the scattering of photon pairs, first we analyze double-excited states of the qubit array, $|\Psi\rangle=\sum_{j_{1} j_{2}} \Psi_{j_{1} j_{2}} b_{j_{1}}^{\dagger} b_{j_{2}}^{\dagger}|0\rangle$. We can obtain the eigenstates and eigenvalues $2 \varepsilon$ by diagonalizing the Hamiltonian Eq. (2). We are interested only in the symmetric boson solutions satisfying $\Psi_{i_{1} i_{2}}=\Psi_{i_{2} i_{1}}$. Because of the qubit-photon interaction, the double-excited state is unstable, and it will decay into a single-excited state and a freely propagating photon. The amplitude of the radiative transition from the double-excited state $|\Psi\rangle$ to a singleexcited state $b_{j}^{\dagger}|0\rangle$ is determined by

$$
d_{j}=\sum_{j^{\prime}} e^{i \omega_{0} z_{j^{\prime}} / c} \Psi_{j j^{\prime}}
$$

According to Fermi's golden rule, the total decay rate is given by the sum of the individual decay rates to all single-excited states, and reads

$$
\Gamma_{1}=\Gamma_{0} \sum_{j}\left|d_{j}\right|^{2}
$$

Such a decay rate determines the imaginary part of the eigenvalues, $\operatorname{Im} \varepsilon=-\Gamma_{1}$. A detailed derivation of Eq. (5) is presented in Supplemental Material [45].

The eigenstates are usually classified, depending on a ratio of their decay rate to that of the individual qubit, as either superradiant $\left(\Gamma_{1} \sim N \Gamma_{0}\right)$, bright $\left(\Gamma_{1} \sim \Gamma_{0}\right)$, or subradiant $\left(\Gamma_{1} \ll \Gamma_{0}\right)$.

However, for double-excited states, this classification is incomplete since it characterizes emission of the first photon only, and it does not provide information about the subsequent emission of the second photon. Here, we characterize the latter process by the amplitude $\sum_{j} d_{j}$ that quantifies the effective dipole moment of the superposition of single-excited states after emission of the first photon. We identify the states for which the amplitudes of individual radiative transitions are finite but out of phase, so that $\sum_{j} d_{j}$ vanishes, as products of a bright state and a subradiant state, and we term them twilight states. The twilight state quickly decays into a single outgoing photon and a single-excited state. However, the latter excitation appears subradiant and the second photon is emitted after a long time $\sim 1 /\left(\varphi^{2} \Gamma_{0}\right)$ with $\varphi \equiv \omega_{0}\left|z_{2}-z_{1}\right| / c$, providing long-lived photon-photon correlations. Namely, the correlation function $g^{(2)}(t)$ has contributions with the lifetime $\left[\sim 1 /\left(\varphi^{2} \Gamma_{0}\right)\right]$, much longer than that of the individual qubits $\left(\sim 1 / \Gamma_{0}\right)$. The contribution of a twilight state combines the features of the subradiant and bright states. While it has weak amplitude $\propto \varphi^{2}$, it can be resonantly excited in a relatively broad spectral range $\sim \Gamma_{0}$ and decays with a small rate $\sim \varphi^{2} \Gamma_{0}$. A detailed analysis is given in Fig. S11 in Ref. [45].

Thus, depending on the magnitude of $\sum_{j}\left|d_{j}\right|^{2}$ and $\left|\sum_{j} d_{j}\right|^{2}$, the double-excited eigenstates can be classified as superradiant, twilight, and subradiant; see Table I. As demonstrated by our calculations, the short-period array of $N>2$ two-level qubits has one superradiant state, $N(N-3) / 2$ subradiant states, and $(N-1)$ twilight states with total energies around $2 \omega_{0}$. Figure 2 shows the dependence of the decay rate for superradiant states (red diamonds), twilight states (green stars), and subradiant 
TABLE I. Classification of the double-excited states depending on the amplitudes of the radiative transition rates $d_{j}$. Illustrations in the upper row sketch the (nonsymmetrized) two-photon wave function.

\begin{tabular}{lccc}
\hline \hline & $\sim N$ & $\sim 1$ & $\ll 1$ \\
\hline$\sum_{j}\left|d_{j}\right|^{2}$ & $\sim N^{2}$ & $\ll 1$ & $\ll 1$ \\
$\left|\sum_{j} d_{j}\right|^{2}$ & $\sim N^{\text {Superradiant }}$ & Subradiant \\
\hline \hline
\end{tabular}

states (black dots and diamonds) on the number of qubits. For the superradiant state, $\Gamma_{1}$ is proportional to $(N-1) \Gamma_{0}$. For most subradiant double-excited states (black diamonds in Fig. 2), $\Gamma_{1}$ becomes smaller by 2 orders of magnitude as the number of qubits increases from $N=3$ to $N=4$, and for $N \geq 4$ satisfies the scaling relation $\Gamma_{1} \sim \Gamma_{0} \varphi^{2} / N^{3}$, where $\varphi=\left(\omega_{0} / c\right)\left|z_{2}-z_{1}\right|$.

In order to understand the threshold of $N=4$ qubits for the two-excitation subradiant states, we consider the radiative decay for the double-excited states in the limiting case where all the qubits are located in the same point, $z_{j} \equiv 0$. The wave function of the subradiant state should satisfy three conditions: (i) $d_{j_{1}}=\sum_{j_{2}} \Psi_{j_{1} j_{2}}=0$ for all $j_{1}$, (ii) the symmetricity $\Psi_{j_{1} j_{2}}=\Psi_{j_{2} j_{1}}$, and (iii) zero diagonal elements, $\Psi_{j_{1} j_{1}}=0$, since we look for the states where neither of the qubits is occupied twice. While these conditions cannot be simultaneously met for the arrays with $N=2$ and $N=3$ qubits, there exist two subradiant states for $N=4$ qubits with

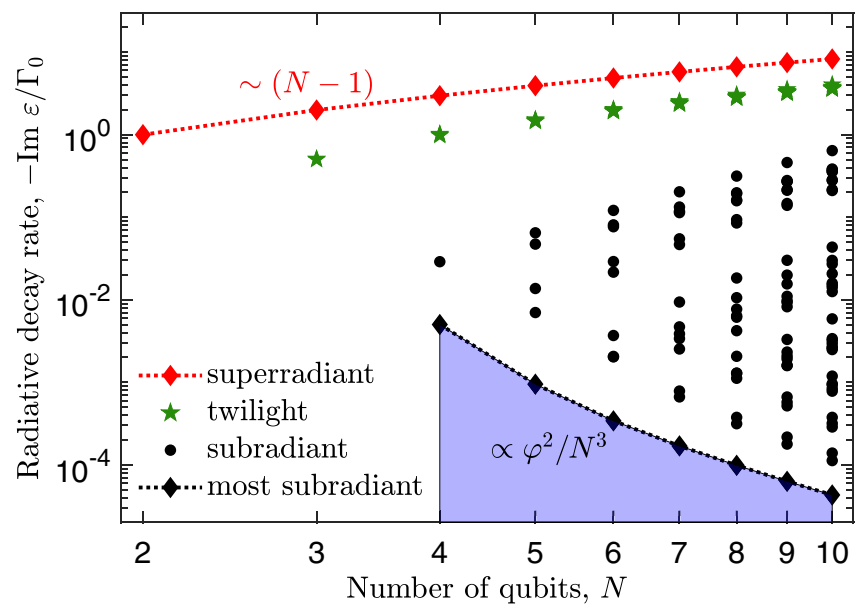

FIG. 2. First-order radiative decay rates of double-excited states depending on the number of qubits in an array $N$. Red diamonds, green stars, black dots, and black diamonds correspond to the superradiant, twilight, subradiant states, and most subradiant states, respectively. Calculation has been performed for $\chi=10^{4} \Gamma_{0}, \varphi \equiv \omega_{0}\left|z_{2}-z_{1}\right| / c=0.1$.

$$
\begin{aligned}
& {\left[\Psi_{i j}\right]_{1}=\frac{\sqrt{2}}{4}\left(\begin{array}{cccc}
0 & 0 & 1 & -1 \\
0 & 0 & -1 & 1 \\
1 & -1 & 0 & 0 \\
-1 & 1 & 0 & 0
\end{array}\right),} \\
& {\left[\Psi_{i j}\right]_{2}=\frac{\sqrt{6}}{12}\left(\begin{array}{cccc}
0 & -2 & 1 & 1 \\
-2 & 0 & 1 & 1 \\
1 & 1 & 0 & -2 \\
1 & 1 & -2 & 0
\end{array}\right),}
\end{aligned}
$$

where the rows and columns represent the coordinate of the first and second excitation, respectively. The first state is just a direct product of the two single-excited subradiant states, $\frac{1}{2}\left(b_{1}^{\dagger}-b_{2}^{\dagger}\right)\left(b_{3}^{\dagger}-b_{4}^{\dagger}\right)|0\rangle$, while the second state has a more intricate structure. Because of the short length of the array, $N=4$, neither of the subradiant states Eq. (6) is described by the fermionic ansatz [34]; see Ref. [45] for more details. When the spacing between the qubits becomes nonzero, $0<\varphi \ll 1$, these subradiant states become slightly bright:

$$
\begin{aligned}
& \varepsilon_{1}=\omega_{0}-\varphi \Gamma_{0}-\frac{i \varphi^{2}}{2} \Gamma_{0}, \\
& \varepsilon_{2}=\omega_{0}-\frac{7 \varphi}{3} \Gamma_{0}-\frac{157 i \varphi^{2}}{54} \Gamma_{0},
\end{aligned}
$$

where the first-order decay rates are proportional to $\varphi^{2} \ll 1$. As such, the subradiant states become optically active and can be probed in the light scattering spectra. More details can be found in Ref. [45].

Incoherent scattering of photon pairs.-Next, we discuss how the photon-photon interactions are affected by the double-excited states. To this end, we consider the incoherent scattering process, where the two incident photons with the energies $\omega_{1}$ and $\omega_{2}$ are scattered inelastically and converted into a pair of photons with the energies $\omega_{1}^{\prime}$ and $\omega_{2}^{\prime}$, so that $\omega_{1}+\omega_{2}=\omega_{1}^{\prime}+\omega_{2}^{\prime}=2 \varepsilon$. Generally, calculation of the scattering is significantly more challenging than that of the double-excited excitations. The reason is that, instead of the reduced problem Eq. (2) describing only the qubit excitations, one needs to consider the full two-particle Hilbert space. Here, we use the rigorous Green function approach, based on the Hamiltonian Eq. (1) with general qubit anharmonicity $\chi$. While our methodology is conceptually similar to that of Ref. [43], it has the advantage of a compact matrix formulation valid for arbitrary spatial arrangement of the qubits. Thus, contrary to other Greenfunction-based techniques [48,49], we are able to obtain a closed-form analytical answer. Namely, the $S$ matrix describing the forward incoherent scattering reads

$$
\begin{aligned}
& S\left(\omega_{1}^{\prime}, \omega_{2}^{\prime} ; \omega_{1}, \omega_{2}\right)=2 \pi i M \delta\left(\omega_{1}+\omega_{2}-\omega_{1}^{\prime}-\omega_{2}^{\prime}\right), \\
& M=-2 i \Gamma_{0}^{2}\left(\frac{c}{L}\right)^{2} \sum_{i, j} s_{i}^{-}\left(\omega_{1}^{\prime}\right) s_{i}^{-}\left(\omega_{2}^{\prime}\right) Q_{i j} s_{j}^{+}\left(\omega_{1}\right) s_{j}^{+}\left(\omega_{2}\right),
\end{aligned}
$$


where $s_{i}^{ \pm}=\sum_{j} G_{i j} e^{ \pm i \omega z_{j} / c}$ is the structure factor for individual incoming (outgoing) photons, $G(\omega)=$ $\left[\omega-H^{(1)}(\omega)\right]^{-1}$ is the single-particle Green function, and $L$ is the normalization length. Here, $Q_{i j}$ is the scattering kernel given by $Q=-i \chi(1-i \chi \Sigma)^{-1}$, where $\Sigma_{i j}(\varepsilon)=$ $\int G_{i j}(\omega) G_{i j}(2 \varepsilon-\omega) d \omega /(2 \pi)$. Equation (8) remains valid in the limit of two-level qubits, $\chi \rightarrow \infty$, when $Q \rightarrow \Sigma^{-1}$.

The result becomes more transparent when the Green function is evaluated in the Markovian approximation as $G(\omega)=\left[\omega-H\left(\omega_{0}\right)\right]^{-1}$. The integration over frequency in $\Sigma_{i j}(\varepsilon)$ can then be carried out analytically, yielding

$$
Q_{i j}=i \chi\left(\frac{2 \varepsilon-H^{(2)}}{H^{(2)}+\mathcal{U}-2 \varepsilon}\right)_{i i, j j} ;
$$

see Supplemental Material [45] for details. Here, the effective two-particle Hamiltonian is given by a sum of individual photon Hamiltonians, $H_{i_{1} i_{2} ; j_{1} j_{2}}^{(2)}=\delta_{i_{2}, j_{2}} H_{i_{1} j_{1}}+\delta_{i_{1}, j_{1}} H_{i_{2} j_{2}}$, and the interaction term, $\mathcal{U}_{i_{1} i_{2} ; j_{1} j_{2}}=\delta_{i_{1} i_{2}} \delta_{j_{1} j_{2}} \delta_{i_{1} j_{1}} \chi$. The difference $2 \varepsilon-H^{(2)}$ in the numerator of Eq. (9) reflects the destructive quantum interference in the two-photon scattering [37,42]. The matrix $Q$ has resonances at the eigenstates $2 \varepsilon$ of the Hamiltonian Eq. (2) in the twoexcitation subspace $H^{(2)}+\mathcal{U}$. In the vicinity of the resonance, $\varepsilon \approx \operatorname{Re} \varepsilon_{\nu}$, Eq. (9) can be simplified to

$$
Q_{i j}(\varepsilon) \approx \frac{2 i \Gamma_{0}^{2} d_{i} d_{j}^{*}}{\operatorname{Re} \varepsilon_{\nu}-i \Gamma_{0} \sum_{j^{\prime}}\left|d_{j^{\prime}}\right|^{2}-\varepsilon},
$$

where we assume $\chi \rightarrow \infty$. The analytical structure of the two-photon kernel $Q$ is now quite clear. The amplitudes of the radiative transitions $d_{j}$ determine both the resonance linewidth in the denominator [which matches the decay rate Eq. (5)] and the effective oscillator strength of the twophoton resonance in the numerator of Eq. (10). This results in the condition $-2 \Gamma_{0} \operatorname{Re} \operatorname{Tr} Q=|\operatorname{Tr} Q|^{2}$ that generalizes the optical theorem to the interacting two-photon case.

The calculated incoherent scattering spectra are summarized in Fig. 3. We present the total forward scattering rate,

$I\left(\omega_{1}, \omega_{2}\right)=\frac{1}{2} \int\left|M\left(\omega_{1}^{\prime}, \omega_{1}+\omega_{2}-\omega_{1}^{\prime} ; \omega_{1}, \omega_{2}\right)\right|^{2} \frac{d \omega_{1}^{\prime}}{2 \pi}$,

integrated over the frequencies of the scattered photons. As such, the scattering map of Fig. 3(c) shows both the resonances when either $\omega_{1}$ or $\omega_{2}$ is tuned to the singleexcited subradiant eigenstates (horizontal and vertical dashed lines), and the two-photon resonances, when the total energy $\omega_{1}+\omega_{2}$ is in resonance with the doubleexcited subradiant state (diagonal solid lines). We show the scattering as a function of $\omega_{1}$ by fixing $\omega_{2}-\omega_{1}=6 \varphi \Gamma_{0}$ and $\omega_{2}-\omega_{1}=0$; see Figs. 3(a) and 3(b), respectively. Two resonant peaks marked by blue arrows are the positions of
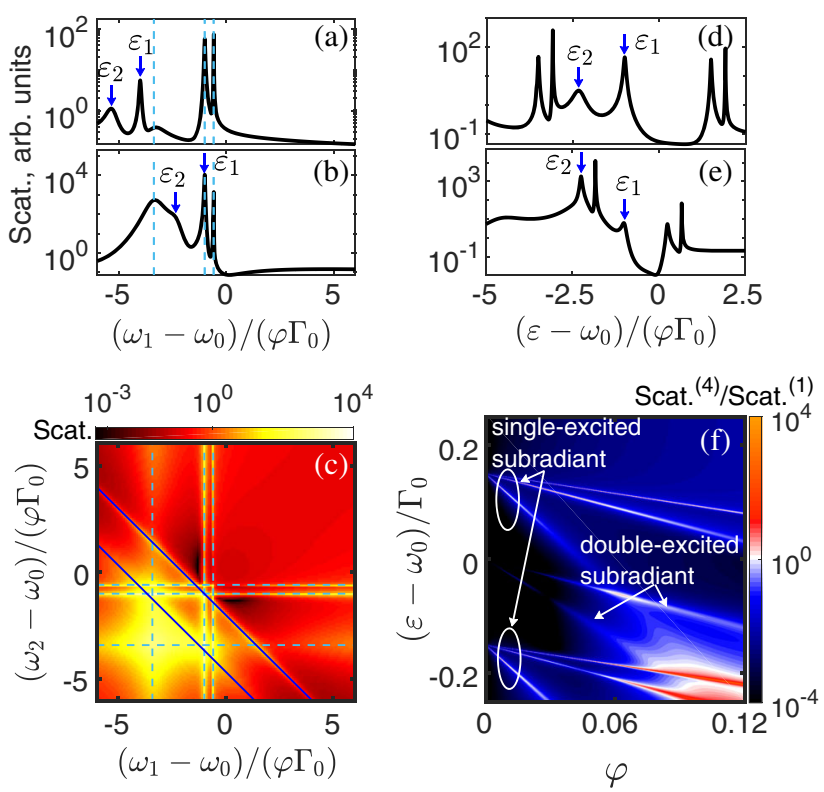

FIG. 3. Incoherent forward scattering intensity for an array of four qubits. Scattering intensity as a function of $\omega_{1}$ for (a) $\omega_{2}-\omega_{1}=6 \varphi \Gamma_{0}$ and (b) $\omega_{2}-\omega_{1}=0$. Thin dashed vertical lines indicate the positions of single-excited eigenmodes. The arrows show double-excited subradiant modes with the energies $\varepsilon_{1,2}$. (c) False-color map of the scattering vs $\omega_{1}$ and $\omega_{2}$. Dashed and solid lines indicate the one- and two-photon resonances, respectively. The other parameters in the left-hand panel are $\chi=10^{4} \Gamma_{0}$, $\varphi \equiv \omega_{0}\left|z_{2}-z_{1}\right| / c=0.1$.(d),(e) Scattering intensity as a function of average energy $\varepsilon$ for given $\varphi=0.06$ and $\varphi=0.12$, respectively. (f) Normalized false-color map of the scattering vs array period $\varphi$ and mean energy of incoming photons $\varepsilon$. The other parameters are $\chi=10^{4} \Gamma_{0}$ and $\omega_{2}-\omega_{1}=0.3 \Gamma_{0}$.

$\varepsilon_{1}$ and $\varepsilon_{2}$, the energies of double-excited subradiant states. The outgoing photon pairs can also have strong spatial correlations depending on the nature of the resonant states [45]. In the considered case of 4 qubits, there is a point in Fig. 3(c) where the vertical, horizontal, and diagonal lines cross over. This triple-resonant condition occurs when one of the single-excited subradiant states has energy with the same real part $\omega_{0}-\varphi \Gamma_{0}$ as that for the double-excited subradiant state Eq. (7). Thus, when both incident photons have the same energies, $\omega_{1}=\omega_{2}=\omega_{0}-\varphi \Gamma_{0}$, the triple resonance further enhances the scattering; see the peak at $\varepsilon_{1}$ in Fig. 3(b).

Figures 3(d)-3(f) show the scattering depending on the array period $\varphi \equiv \omega_{0}\left|z_{2}-z_{1}\right| / c$. The spectra are calculated for fixed detuning $\omega_{2}-\omega_{1}=0.3 \Gamma_{0}$ and are normalized to the maximum of total forward scattering for a single qubit, $N=1$. The dark region around $\varepsilon=\omega_{0}, \varphi=0$ in Fig. 3(f) reflects that the scattering is suppressed by the destructive quantum interference $[28,42]$, which also exists in $N=2,3$ qubits [45]. However, due to subradiant resonances emerging for $\varphi>0$, the scattering for $N=4$ can exceed that for $N=1$ by several orders of magnitude. The single- and double-excited subradiant resonances can be traced by their 
different dependence on $\varphi$. The scattering reaches a local maximum at double-resonant conditions. Namely, either a single-excited and a double-excited resonance or two single-excited resonances can occur simultaneously; see the spectra in Figs. 3(d) and 3(e), respectively.

Multiexcited states.-The considered subradiant states are not limited to double excitations. We expect even richer physics for the excitations with a higher number of photons, $M>2$, which can already be accessed experimentally [50]. As $M$ increases, a threshold of the qubit number for subradiant states will also change. To reveal how the subradiant state depends on the excitation number $M$ and qubit number $N$, we find the eigenstate with the energy around $M \omega_{0}$ that has the minimal decay rate for different $M$ and $N$ [45]. The threshold, determined by the decrease of $\Gamma_{1}$ down to $\sim \varphi^{2} \Gamma_{0}$, occurs for $N=2 M$. The notion of metastable twilight states can also be extended in the general $M$-body case: $M^{\prime}$ particles in bright (or even superradiant) states multiplied by $M-M^{\prime}$ subradiant states. How these multiexcited states affect the incoherent $M$-photon scattering is beyond the scope of this Letter.

Conclusion.-We believe that our results open a new research direction for harnessing light-matter interactions in quantum photonics. In particular, the subradiant states boost the incoherent scattering while the twilight states perpetuate the photon-photon correlations. Custom-tailored long-lived entangled photons could be employed for storage and processing of quantum information.

We acknowledge useful discussions with J. Brehm, I. Iorsh, A. V. Kavokin, E. Redchenko, A. A. Sukhorukov, A. V. Ustinov, and V. I. Yudson. This work was supported by the Australian Research Council. C. L. was supported by the National Natural Science Foundation of China (NNSFC) (Grants No. 11874434 and No. 11574405). Y. K. was partially supported by the International Postdoctoral Exchange Fellowship Program (Grant No. 20180052). A. V.P. acknowledges the support from the Russian Science Foundation (Project No. 19-72-00080). A. N. P. has been partially supported by the Russian Foundation for Basic Research Grant No. 18-29-20037MK.

*lichaoh2@mail.sysu.edu.cn poddubny@coherent.ioffe.ru

[1] D. E. Chang, V. Vuletić, and M. D. Lukin, Quantum nonlinear optics-Photon by photon, Nat. Photonics 8, 685 (2014).

[2] K. E. Dorfman, F. Schlawin, and S. Mukamel, Nonlinear optical signals and spectroscopy with quantum light, Rev. Mod. Phys. 88, 045008 (2016).

[3] S. Kruk, A. Poddubny, D. Smirnova, L. Wang, A. Slobozhanyuk, A. Shorokhov, I. Kravchenko, B. LutherDavies, and Y. Kivshar, Nonlinear light generation in topological nanostructures, Nat. Nanotechnol. 14, 126 (2019).
[4] H. J. Kimble, The quantum internet, Nature (London) 453, 1023 (2008).

[5] A. Tittl, A. Leitis, M. Liu, F. Yesilkoy, D.-Y. Choi, D. N. Neshev, Y.S. Kivshar, and H. Altug, Imaging-based molecular barcoding with pixelated dielectric metasurfaces, Science 360, 1105 (2018).

[6] R. H. Dicke, Coherence in spontaneous radiation processes, Phys. Rev. 93, 99 (1954).

[7] D. Roy, C. M. Wilson, and O. Firstenberg, Colloquium: Strongly interacting photons in one-dimensional continuum, Rev. Mod. Phys. 89, 021001 (2017).

[8] D. E. Chang, J. S. Douglas, A. González-Tudela, C.-L. Hung, and H. J. Kimble, Colloquium: Quantum matter built from nanoscopic lattices of atoms and photons, Rev. Mod. Phys. 90, 031002 (2018).

[9] A. F. Kockum, A. Miranowicz, S. D. Liberato, S. Savasta, and F. Nori, Ultrastrong coupling between light and matter, Nat. Rev. Phys. 1, 19 (2019).

[10] E. L. Ivchenko, A. I. Nesvizhskii, and S. Jorda, Bragg reflection of light from quantum-well structures, Phys. Solid State 36, 1156 (1994).

[11] G. Birkl, M. Gatzke, I. H. Deutsch, S. L. Rolston, and W. D. Phillips, Bragg Scattering from Atoms in Optical Lattices, Phys. Rev. Lett. 75, 2823 (1995).

[12] R. G. DeVoe and R. G. Brewer, Observation of Superradiant and Subradiant Spontaneous Emission of Two Trapped Ions, Phys. Rev. Lett. 76, 2049 (1996).

[13] A. Chumakov, L. Niesen, D. Nagy, and E. Alp, Nuclear resonant scattering of synchrotron radiation by multilayer structures, Hyperfine Interact. 123, 427 (1999).

[14] J. Hendrickson, B. C. Richards, J. Sweet, G. Khitrova, A. N. Poddubny, E. L. Ivchenko, M. Wegener, and H. M. Gibbs, Excitonic polaritons in Fibonacci quasicrystals, Opt. Express 16, 15382 (2008).

[15] D. Goldberg, L. I. Deych, A. A. Lisyansky, Z. Shi, V. M. Menon, V. Tokranov, M. Yakimov, and S. Oktyabrsky, Exciton-lattice polaritons in multiple-quantum-well-based photonic crystals, Nat. Photonics 3, 662 (2009).

[16] A. F. van Loo, A. Fedorov, K. Lalumiere, B. C. Sanders, A. Blais, and A. Wallraff, Photon-mediated interactions between distant artificial atoms, Science 342, 1494 (2013).

[17] A. Poddubny and E. Ivchenko, Resonant diffraction of electromagnetic waves from solids (a review), Phys. Solid State 55, 905 (2013).

[18] J. A. Mlynek, A. A. Abdumalikov, C. Eichler, and A. Wallraff, Observation of Dicke superradiance for two artificial atoms in a cavity with high decay rate, Nat. Commun. 5, 5186 (2014).

[19] W. Guerin, M. O. Araújo, and R. Kaiser, Subradiance in a Large Cloud of Cold Atoms, Phys. Rev. Lett. 116, 083601 (2016).

[20] S. D. Jenkins, J. Ruostekoski, N. Papasimakis, S. Savo, and N. I. Zheludev, Many-Body Subradiant Excitations in Metamaterial Arrays: Experiment and Theory, Phys. Rev. Lett. 119, 053901 (2017).

[21] M. F. Limonov, M. V. Rybin, A. N. Poddubny, and Y. S. Kivshar, Fano resonances in photonics, Nat. Photonics 11, 543 (2017).

[22] P. Wolf, S. C. Schuster, D. Schmidt, S. Slama, and C. Zimmermann, Observation of Subradiant Atomic 
Momentum States with Bose-Einstein Condensates in a Recoil Resolving Optical Ring Resonator, Phys. Rev. Lett. 121, 173602 (2018).

[23] P. Weiss, M. O. Araújo, R. Kaiser, and W. Guerin, Subradiance and radiation trapping in cold atoms, New J. Phys. 20, 063024 (2018).

[24] Z. Wang, H. Li, W. Feng, X. Song, C. Song, W. Liu, Q. Guo, X. Zhang, H. Dong, D. Zheng, H. Wang, and D.-W. Wang, Generation and controllable switching of superradiant and subradiant states in a 10-qubit superconducting circuit, arXiv:1907.13468.

[25] L. Carletti, K. Koshelev, C. De Angelis, and Y. Kivshar, Giant Nonlinear Response at the Nanoscale Driven by Bound States in the Continuum, Phys. Rev. Lett. 121, 033903 (2018).

[26] A. N. Poddubny and D. A. Smirnova, Nonlinear generation of quantum-entangled photons from high-Q states in dielectric nanoparticles, arXiv:1808.04811.

[27] K. Koshelev, S. Kruk, J.-H. Choi, E. V. Melik-Gaykazyan, D. Smirnova, H.-G. Park, and Y. Kivshar, Observation of extraordinary SHG from all-dielectric nanoantennas governed by bound states in the continuum, in Proceedings of the Conference on Lasers Electro-Optics (Optical Society of America, Washington, DC, 2019), p. FW4B.3.

[28] V. Yudson and V. Rupasov, Exact Dicke superradiance theory: Bethe wavefunctions in the discrete atom model, Sov. Phys. JETP 59, 478 (1984).

[29] J.-T. Shen and S. Fan, Strongly correlated multiparticle transport in one dimension through a quantum impurity, Phys. Rev. A 76, 062709 (2007).

[30] J.-T. Shen and S. Fan, Strongly Correlated Two-Photon Transport in a One-Dimensional Waveguide Coupled to a Two-Level System, Phys. Rev. Lett. 98, 153003 (2007).

[31] Y.-L. L. Fang and H. U. Baranger, Waveguide QED: Power spectra and correlations of two photons scattered off multiple distant qubits and a mirror, Phys. Rev. A 91, 053845 (2015).

[32] A. Albrecht, L. Henriet, A. Asenjo-Garcia, P. B. Dieterle, O. Painter, and D. E. Chang, Subradiant states of quantum bits coupled to a one-dimensional waveguide, New J. Phys. 21, 025003 (2019).

[33] G. Calajó, Y.-L. L. Fang, H. U. Baranger, and F. Ciccarello, Exciting a Bound State in the Continuum through Multiphoton Scattering Plus Delayed Quantum Feedback, Phys. Rev. Lett. 122, 073601 (2019).

[34] Y.-X. Zhang and K. Mølmer, Theory of Subradiant States of a One-Dimensional Two-Level Atom Chain, Phys. Rev. Lett. 122, 203605 (2019).

[35] L. Henriet, J. S. Douglas, D. E. Chang, and A. Albrecht, Critical open-system dynamics in a one-dimensional optical-lattice clock, Phys. Rev. A 99, 023802 (2019).

[36] Y.-X. Zhang, C. Yu, and K. Mølmer, Subradiant dimer excited states of atom chains coupled to a 1D waveguide, arXiv:1908.01818.
[37] V. I. Yudson and P. Reineker, Multiphoton scattering in a one-dimensional waveguide with resonant atoms, Phys. Rev. A 78, 052713 (2008).

[38] O. Firstenberg, T. Peyronel, Q.-Y. Liang, A. V. Gorshkov, M. D. Lukin, and V. Vuletić, Attractive photons in a quantum nonlinear medium, Nature (London) 502, 71 (2013).

[39] M. Laakso and M. Pletyukhov, Scattering of Two Photons from Two Distant Qubits: Exact Solution, Phys. Rev. Lett. 113, 183601 (2014).

[40] Y.-L. L. Fang, H. Zheng, and H. U. Baranger, Onedimensional waveguide coupled to multiple qubits: Photon-photon correlations, EPJ Quantum Technol. 1, 3 (2014).

[41] S. Xu and S. Fan, Input-output formalism for few-photon transport: A systematic treatment beyond two photons, Phys. Rev. A 91, 043845 (2015).

[42] A. Muthukrishnan, G. S. Agarwal, and M. O. Scully, Inducing Disallowed Two-Atom Transitions with Temporally Entangled Photons, Phys. Rev. Lett. 93, 093002 (2004).

[43] H. Zheng and H. U. Baranger, Persistent Quantum Beats and Long-Distance Entanglement from WaveguideMediated Interactions, Phys. Rev. Lett. 110, 113601 (2013).

[44] A. V. Poshakinskiy and A. N. Poddubny, Biexcitonmediated superradiant photon blockade, Phys. Rev. A 93, 033856 (2016).

[45] See Supplemental Material at http://link.aps.org/ supplemental/10.1103/PhysRevLett.123.253601 for details of (S1) the effective model for the excitations, (S2) the decay process, (S3) subradiant states, (S4) two-photon scattering, (S5) the threshold of the qubit number for subradiant states, and (S6) subradiant and twilight states in photon-photon correlations, which includes Refs. [32,34,36,44,46,47].

[46] V. Kashcheyevs and B. Kaestner, Universal Decay Cascade Model for Dynamic Quantum Dot Initialization, Phys. Rev. Lett. 104, 186805 (2010).

[47] E. L. Ivchenko, Optical Spectroscopy of Semiconductor Nanostructures (Alpha Science International, Harrow, England, 2005).

[48] Ş. E. Kocabaş, Effects of modal dispersion on few-photonqubit scattering in one-dimensional waveguides, Phys. Rev. A 93, 033829 (2016).

[49] M. P. Schneider, T. Sproll, C. Stawiarski, P. Schmitteckert, and K. Busch, Green's-function formalism for waveguide QED applications, Phys. Rev. A 93, 013828 (2016).

[50] Q.-Y. Liang, A. V. Venkatramani, S. H. Cantu, T. L. Nicholson, M. J. Gullans, A. V. Gorshkov, J. D. Thompson, C. Chin, M. D. Lukin, and V. Vuletić, Observation of threephoton bound states in a quantum nonlinear medium, Science 359, 783 (2018). 